\title{
A note about entropy and inconsistency in evidence theory
}

\author{
Anne-Laure Jousselme ${ }^{1}$, Frédéric Pichon ${ }^{2}$, Nadia Ben Abdallah ${ }^{3}$, and \\ Sébastien Destercke ${ }^{4}$ \\ 1 NATO STO Centre for Maritime Research and Experimentation, La Spezia, Italy \\ anne-laure.jousselme@cmre.nato.int \\ 2 Univ. Artois, UR 3926 LGI2A, F-62400 Béthune, France \\ frederic.pichon@univ-artois.fr \\ 3 Independent researcher, Clamart, France \\ nadia.ben. abdallah7@gmail.com \\ 4 Univ. Technologie de Compiègne, UMR CNRS 7253 Heudiasyc, Compiègne, France \\ sebastien.destercke@hds.utc.fr
}

\begin{abstract}
Information content is classically measured by entropy measures in probability theory, that can be interpreted as a measure of internal inconsistency of a probability distribution. While extensions of Shannon entropy have been proposed for quantifying information content of a belief function, other trends have been followed which rather focus on the notion of consistency between sets. Relying on previous general entropy measures of probability, we propose in this paper to establish some links between the different measures of internal inconsistency of a belief functions. We propose a general formulation which encompasses inconsistency measures derived from Shannon entropy as well as those derived from the $N$-consistency family of measures.
\end{abstract}

Keywords: Information content · Inconsistency · Conflict · Entropy

\section{Introduction}

In a multi-intelligence context, information generally arises from different systems (or services), each having their own local representation and underlying mathematical formalism. The choice of this formalism is usually driven by the nature of data or information to be handled. For instance, numerical data (when available in large volume) usually summarize in probabilistic models, while human judgments are best handled by logical approaches managing knowledge bases. The underlying mathematical setting constrains not only the internal reasoning of those services, but also their output which includes some metainformation such as the information content or information value. In decision support, conflict or inconsistency measures play an essential role in detecting sources' defect or intentional deception, but also in quantifying information credibility when no ground truth is available. In probability theory, measuring conflict goes back to Shannon entropy [16] which quantifies an inverse notion of 
the information contained in a probability distribution, i.e. a notion of internal inconsistency (e.g., [10]). Indeed, the state of maximum inconsistency is reached by the uniform distribution while the state of minimal inconsistency is reached whenever an element is assigned a probability of 1 . In propositional logic, a belief base (a set of formulas) is inconsistent if it entails the contradiction ${ }^{5}[6]$. In evidence theory, which captures both probabilistic and logical notions, measuring inconsistency of belief functions has thus naturally followed two main trends: on the one hand, some measures extend Shannon entropy (e.g., $[21,5,9,8,7])$ and on the other hand inconsistency is measured through the inconsistency between sets (e.g., $[4,3,13])$.

We propose in this paper to establish some links between the different approaches to inconsistency measurement. In Section 2, we introduce basic concepts and notations of belief functions together with two families of measures of entropy for probabilities. In Section 3 we survey the different trends followed and propose a general formulation, and highlight the main elementary constructs leading to inconsistency measures. In Section 4, after revealing the "hidden" mass of the empty set within Shannon entropy we propose a general formulation which encompasses most of classical existing measures across the different approaches. We conclude in Section 5 on perspectives and future work.

\section{Background and notations}

\subsection{Belief functions}

We consider in this paper the singular interpretation of belief functions as developed by Shafer [15] and Smets and Kennes [17]. Belief functions are thus used to represent and handle subjective uncertainty (or beliefs) of an agent about the actual state of the world. Let us denote by $X$ an uncertain variable defined on frame of discernment $\mathcal{X}=\left\{x_{1}, \ldots, x_{K}\right\}$ representing the possible values (states) for that variable. A mass function is a mapping $m: 2^{\mathcal{X}} \rightarrow[0,1]$ satisfying $\sum_{A \subset \mathcal{X}} m(A)=1$. The mass $m(A)$ represents the amount of belief allocated to the fact of knowing only that $\boldsymbol{x} \in A$. We will denote by $\mathcal{M}$ the set of all mass functions on $\mathcal{X}$. Subsets $A$ of $\mathcal{X}$ such that $m(A)>0$ are called focal sets of $m$, and the set of focal sets of $m$ will be denoted by $\mathcal{F}$. A mass function $m$ is called categorical if $m(A)=1$ for some $A \subseteq \mathcal{X}$, in which case it defines a classical set and will be denoted by $m_{A}$ in the following. It is called vacuous if $m(\mathcal{X})=1$ and denoted by $m_{\mathcal{X}}$. It represents total ignorance. The mass function is called empty if $m(\emptyset)=1$ and denoted by $m_{\emptyset}$. It represents total inconsistency in the agent's beliefs about the set of values that are conceivable for $\boldsymbol{x}$ [18]. It is called $B$ ayesian if $m(A) \neq 0$ only for $|A|=1$ and defines a probability distribution. And finally it is called normalised if $m(\emptyset)=0$.

We define a consistency index between two sets to satisfy minimally:

$$
\phi(A, B)=\left\{\begin{array}{l}
0 \text { if } A \cap B=\emptyset \\
1 \text { if } A=B
\end{array}\right.
$$

\footnotetext{
${ }^{5}$ Or equivalently, is unsatisfiable or has no model.
} 
Equivalent representations of a mass function $m$ are the belief function Bel and the plausibility function $\mathrm{Pl}$ which follow the general formulation:

$$
f(A)=\sum_{B \subseteq \mathcal{X}} m(B) \phi(A, B)
$$

$\operatorname{Pl}(A)$ is obtained with $\phi(A, B)=1$ if $A \cap B \neq \emptyset$ and 0 otherwise, and is the amount of belief consistent with $\boldsymbol{x} \in A ; \operatorname{Bel}(A)$ is obtained with $\phi(A, B)=1$ if $B \subseteq A$ and 0 otherwise, and is the amount of belief implying $\boldsymbol{x} \in A$. The contour function $\pi: \mathcal{X} \rightarrow[0,1]$ is such that $\pi(x)=\operatorname{Pl}(\{x\})$, for all $x \in \mathcal{X}$. It is the plausibility function restricted to the singletons of $\mathcal{X}$.

Let $m_{1}$ and $m_{2}$ be two mass functions representing pieces of evidence about $\boldsymbol{x}$. Their combination by the conjunctive rule [2] is defined by, for all $A \subseteq \mathcal{X}$,

$$
m_{1} \cap_{2}(A)=\sum_{B \cap C=A} m_{1}(B) m_{2}(C) .
$$

The conflict between $m_{1}$ and $m_{2}$ can be quantified as $m_{1} \bigcap_{2}(\emptyset)$ [15].

\subsection{Generalized entropy measures of probabilities}

Rényi entropy Given a probability distribution $p$ over $\mathcal{X}$, the Rényi entropy of order $\alpha$, is defined for a parameter $\alpha \in \mathbb{R}^{+} \backslash\{1\}$ as [14]:

$$
\delta_{R}^{(\alpha)}(p)=\frac{1}{1-\alpha} \log \left(\sum_{x \in \mathcal{X}} p(x)^{\alpha}\right)
$$

For $\alpha=0,(4)$ is Hartley measure $\log (|\mathcal{X}|)$, while Shannon entropy is retrieved whenever $\alpha \rightarrow 1, \delta_{R}^{(1)}(p)=-\sum_{x \in \mathcal{X}} p(x) \log p(x)$. For $\alpha=2$, the collision entropy is defined, $\delta_{R}^{(2)}(p)=-\sum_{x \in \mathcal{X}} p(x)^{2}$. Interestingly, $\delta_{R}^{(\alpha)}(p)$ is a decreasing function of $\alpha$, and for $\alpha \rightarrow+\infty$, we obtain the minimum entropy, $\delta_{R}^{(\infty)}(p)=-\log \max _{x \in \mathcal{X}} p(x)$.

Power entropy Another family of entropy measures which still extends Shannon entropy has been defined by Vajda and Zvárová [20], relying on the decreasing power function $\psi_{a}:[0 ; 1] \rightarrow \mathbb{R}$, for $a \in \mathbb{R}$ :

$$
\psi_{a}(\mu)=\left\{\begin{array}{l}
\frac{1}{a-1}\left(1-\mu^{a-1}\right), \text { if } a \neq 1 \\
-\log (\mu) \text { if } a=1
\end{array}\right.
$$

with $\psi_{a}(0)=\lim _{\mu \rightarrow 0} \psi_{a}(\mu)$ if $a \neq 1, \psi_{1}(0)=+\infty$ and $0 \cdot \psi_{a}(0)=0$. Power entropy measures are thus defined as [20]:

$$
\delta_{V}^{(a)}(p)=\sum_{x \in \mathcal{X}} p(x) \psi_{a}(p(x))
$$


Similarly to Rényi entropy, Shannon entropy is obtained for $a=1$ while $\delta_{V}^{(0)}(p)=$ $\log (|\mathcal{X}|)-1$ is one-to-one related to Hartley entropy. We note that for $a \neq 1$ in Eq. (5), Eq. (6) defines Tsallis entropy [19].

Power functions $\psi_{a}$ are interesting as they allow to reverse a consistency notion into an inconsistency notion. Indeed, they are decreasing and satisfy $\psi_{a}(1)=0$, meaning that if $p(x)$ is a degree of consistency, $\psi_{a}(p(x))$ is a degree of inconsistency. In the following, we will denote by $\phi$ indexes or measures of consistency between sets or of a mass function, while the corresponding inconsistency indexes or measures will be denoted by $\delta$. For instance, an inconsistency index corresponding to (1) would satisfy minimally $\delta(A, B)=1$ if $A \cap B=\emptyset$ and 0 if $A=B$.

\section{Inconsistency of belief functions}

\subsection{An entropy approach}

The first trend followed to quantify the internal inconsistency of a belief function, aims at extending Shannon entropy, focusing on the probabilistic dimension of belief functions. Developed mostly between 1982 and 1992, the measures follow the general formulation:

$$
\delta(m)=\sum_{A \subseteq X} m(A)\left(-\log \sum_{B \subseteq X} m(B) \phi(A, B)\right)
$$

where $\phi(A, B)$ is a consistency index between the sets $A$ and $B$ satisfying (1). In particular, Yager's dissonance measure is obtained for $\phi(A, B)=1$ if $A \cap B \neq \emptyset$. Other definitions for $\phi$ still satisfying (1) lead to the measures of confusion from Höhle [5], from Nguyen [12], of discord from Klir \& Ramer [9] and of strife from Klir \& Parviz [8]. All these measures degenerate to Shannon entropy when $m$ is a Bayesian mass function and to Hartley measure when $m$ is categorical.

\subsection{A consistency approach}

Another trend followed has given up on the extension of Shannon and Hartley measures, and their additivity property. Yager first defined a measure of consistency [22] while George and Pal defined the measure of total conflict [4] based on Jaccard index. These two measures correspond to the general formulation:

$$
\delta(m)=\sum_{A \subseteq X} m(A)\left(1-\sum_{B \subseteq X} m(B) \phi(A, B)\right)
$$

\subsection{A $N$-consistency approach}

Recent work [13] proposes the measure of consistency of $m$ :

$$
\phi_{N}(m)=1-m^{(N)}(\emptyset),
$$


where $m^{(N)}$ denotes the mass function resulting from the combination of $m$ by itself $N$ times, i.e. $m^{(N)}=m^{(N-1)} \cap m$ with $m^{(0)}:=m_{\mathcal{X}}$. Hence, we have $m^{(1)}=m, m^{(2)}=m \cap m$ and more generally $m^{(N)}=\bigcap_{1}^{N} m \cdot \phi_{N}(m)$ measures different "shades" of internal consistency of $m$ as $N$ varies and in particular $\phi_{N}(m)$ encompasses two forms of consistency already defined in the literature $[13]$ :

$$
\begin{aligned}
& \phi_{1}(m)=1-m(\emptyset)=\max _{A \subseteq \mathcal{X}} \operatorname{Pl}(A) \\
& \phi_{2}(m)=1-m^{(2)}(\emptyset)=\sum_{A \subseteq \mathcal{X}} m(A) \operatorname{Pl}(A)
\end{aligned}
$$

where $\phi_{1}$ is the measure of so-called probabilistic consistency defined in [3] and $\phi_{2}$ is the measure of consistency defined in [22]. It has been proved as well that $\phi_{|\mathcal{F}|}$ is an alternative measure of logical consistency to the one proposed in [3] as $\phi_{\pi}=\max _{x \in \mathcal{X}} \pi(x)$. More details can be found in [13].

All measures introduced in this section are built upon a measure of consistency between sets, $\phi$ and other elementary constructs such as a reverse function transforming the notion of consistency into inconsistency. Entropy-like measures (Section 3.1) as well as consistency-like measures (Section 3.2) are all based on pair-wise measures of consistency between sets. Instead, the $N$-consistency derived measures (Section 3.3) are based on $N$-wise measures. In the following section 4, we will thus exploit that extension in order to establish a more general formulation covering the three types of approaches.

\section{Extending inconsistency}

Let us introduce the consistency index between $N$ sets as [13]:

$$
\phi_{N}\left(A_{1}, \ldots, A_{N}\right)=\left\{\begin{array}{l}
1 \text { if } \bigcap_{i=1, \ldots, N} A_{i} \neq \emptyset \\
0 \text { else }
\end{array}\right.
$$

and we explore below the extension from pair-wise index to $N$-wise index in measuring the inconsistency of $m$.

\subsection{Observation with probabilities}

Let us start by clarifying why Shannon entropy actually quantifies a notion of internal conflict (or inconsistency). Introduced by Shannon as a measure of information [16], the entropy of a probability distribution is the expected information where $I_{X}(x)=-\log (p(x))$ is the self-information associated with the outcome $x \in \mathcal{X}$. If $p(x)=0$, then $I_{X}(x)=-\infty$ and if $p(x)=1$ then $I_{X}(x)=0$. With $0 \cdot \log 0=0, \delta_{\mathrm{Sh}}(p)=0$ if and only if the distribution is focused on a single element of $\mathcal{X}$ (i.e., it exists one $x$ such that $p(x)=1$ ), and $\delta_{\mathrm{Sh}}(p)=\log (|\mathcal{X}|)$ if and only if $p$ is uniformly distributed over $\mathcal{X}$ (i.e., $p(x)=\frac{1}{|\mathcal{X}|} \forall x \in \mathcal{X}$ ). Hence, $p$ is the most informative when its entropy is null and it is the least informative 
when its entropy is maximum. As such, as noticed for instance in [11,1], Shannon entropy is rather a measure of uncertainty, and even a measure of internal conflict (or inconsistency) for $p$. Indeed, when $p$ is uniformally distributed over $\mathcal{X}$ the internal inconsistency of $p$ is maximum since the same confidence is assigned to inconsistent hypotheses $x_{i}$ of $\mathcal{X}$, i.e. such that $\delta\left(x_{i}, x_{j}\right)=0$ for all $i \neq j$ and $\delta\left(x_{i}, x_{i}\right)=1$ for all $i$, where $\delta$ is an inconsistency index satisfying the properties mentioned in Section 2. We can thus re-write Shannon entropy making apparent the consistency index:

$$
\delta_{R}^{(1)}(p)=\sum_{x \in \mathcal{X}} p(x)\left(-\log \sum_{y \in \mathcal{X}} p(y) \phi(x, y)\right)
$$

Computing the conjunctive self-combination (using (3) for Bayesian mass functions) of $p$ makes it more obvious:

$$
(p @ p)(\emptyset)=p^{(2)}(\emptyset)=1-\sum_{x \in \mathcal{X}} p(x) \sum_{y \in \mathcal{X}} p(y) \phi(x, y)=1-\sum_{x \in \mathcal{X}} p(x)^{2}
$$

which is clearly a measure of the internal inconsistency of $p$, as $p^{(2)}(\emptyset)=0$ iff $\exists x \in \mathcal{X}$ such that $p(x)=1$ and it is maximum for the uniform distribution. Actually, (14) is Gini impurity that can also be written as $\sum_{x \in \mathcal{X}} p(x) .(1-p(x))$. If we denote by $p^{(N)}$ the conjunctive combination of $p$ with itself $N$ times we obtain before any normalisation:

$$
p^{(N)}(\emptyset)=1-\sum_{x \in \mathcal{X}} p(x)^{N}
$$

which is also an inconsistency measure such that $p^{(N)}(\emptyset) \geq p^{(M)}(\emptyset)$ if $N>M$. For integer values of $\alpha$ (that we denote by $N$ ), Rényi entropy in Eq. (4) can thus be written as:

$$
\delta_{R}^{(N)}(p)=-\log \left(1-p^{(N)}(\emptyset)\right)^{\frac{1}{N-1}}
$$

where $\left(1-p^{(N)}(\emptyset)\right)^{\frac{1}{N-1}}$ is a measure of consistency for $p$, and $N \in \mathbb{N}^{*}$.

While for Bayesian mass functions, the $N$-wise comparison of focal sets reduces to the pair-wise comparison, it is not true in the general case that we will detail in the next section.

\subsection{Extension to belief functions}

Let us now introduce the function $\phi_{m}^{(N)}(A)$ which measures the consistency of $m$ relatively to a specific set $A$ of $\mathcal{X}$, so that for $N>1$ :

$$
\phi_{m}^{(N)}(A)=\sum_{B_{1} \subseteq \mathcal{X}} m\left(B_{1}\right) \ldots \sum_{B_{N-1} \subseteq \mathcal{X}} m\left(B_{N-1}\right) \phi_{N}\left(A, B_{1}, \ldots, B_{N-1}\right)
$$


and $\phi_{m}^{(1)}(A)=\phi_{1}(A)$ as defined in Eq. (12). Note that for $N=2$ we get $\phi_{m}^{(2)}(A)=$ $\operatorname{Pl}(A)$. Then, we define the total consistency of $m$ as:

$$
\phi^{(N)}(m)=\sum_{A \subseteq \mathcal{X}} m(A) \phi_{m}^{(N)}(A)
$$

which is the expectation of the local inconsistency of $m$. or $N=1$, we get the probabilistic consistency [3] (Eq. (10)), while for $N=2$ we get Yager's consistency measure [22] (Eq. (11)). We thus propose the following general formulation:

$$
\delta_{a}^{(N)}(m)=\sum_{A \subseteq \mathcal{X}} m(A) \psi_{a}\left(\phi_{m}^{(N)}(A)\right)
$$

where $\psi_{a}$ is the power function introduced in (5).

For $a=1$ (i.e., $-\log ($.$) as inverse function) and N=2$ (i.e., pair-wise comparison of focal sets), we retrieve most of the entropy measures introduced earlier, with different consistency indexes $\phi$ between sets. For $a=2$ (i.e., $1-($. as inverse function) and still $N=2$, we retrieve the consistency-like measures of George and Pal [4], and Yager [22].

Interestingly, this expression allows also capturing the $N$-consistency approaches focused on the mass of the empty set with $N$-wise comparison of focal sets. Indeed, if we consider now the case $a=2$, with a general value of $N,(19)$ becomes simply:

$$
\delta_{2}^{(N)}(m)=1-\phi_{N}(m)=m^{(N)}(\emptyset)
$$

As recalled in Section 3.3, the function $\phi_{|\mathcal{F}|}(m)$ obtained for $N=|\mathcal{F}|$, the number of focal sets of $m$, has been proven to satisfy required properties of a logical consistency of $m$ according to the axioms of [3], qualifying itself thus as a valid alternative measure of logical consistency to $\phi_{\pi}(m)=\max _{x \in \mathcal{X}} \pi(x)$.

Table 1. Entropy and inconsistency measures encompassed by the general expression of Eq. (19), with different values of $a, N$ and $\phi$.

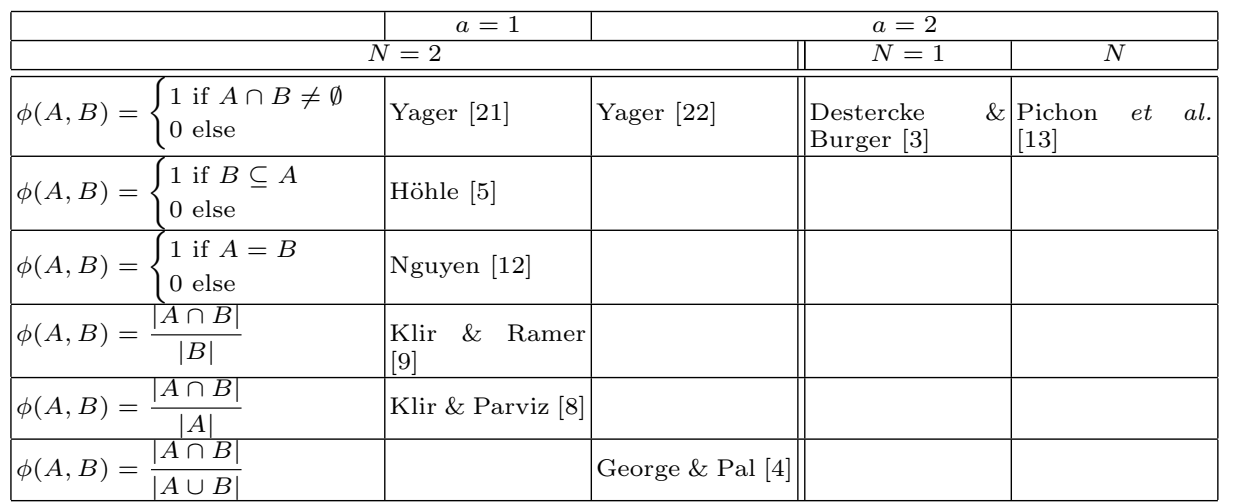


Table 1 summarises the list of measures corresponding to the general expression from Eq. 19 for different values of parameters $a$ and $N$, and for several consistency indices $\phi$ satisfying (1) discussed in this paper. For clarity, the consistency indices are provided for $N=2$ (pair-wise comparison of sets) which corresponds to the most populated case, as displayed in the left part of the table. Other cases for $N=1$ and general $N$ are displayed in the right part of the table, while the corresponding indices are not defined.

We have thus shown that the expression (19) encompasses not only classical entropy measures in evidence theory, but also some non-additive measures of internal conflict and consistency, and last but not the least, measures of inconsistency derived from the $N$-consistency family of measures.

\section{Conclusions}

In this paper, we have firstly shown that most of inconsistency measures defined so far in evidence theory satisfy a general formulation involving a pairwise consistency index between sets, an inverse function transforming the notion of consistency into inconsistency and some expectation operator. Furthermore, by rendering apparent the underlying inconsistency in Rényi entropy family of measures, we have shown how the inconsistency measure derived from the $\mathrm{N}$ consistency falls also under this general formulation. This preliminary result offers new perspectives on the coherent measurement of inconsistency within and across artificial intelligence systems. In future work, we will study other types of inconsistency indexes as well as possible links with other logical consistency and entropy measures. We will also explore the possible orders induced by such information measures.

\section{References}

1. Bronevich, A., Klir, G.J.: Measures of uncertainty for imprecise probabilities: An axiomatic approach. International Journal of Approximate Reasoning 51(4), 365$390(2010)$

2. Dempster, A.P.: Upper and lower probabilities induced by a multivalued mapping. Ann. Math. Stat. 38, 325-339 (1967)

3. Destercke, S., Burger, T.: Toward an axiomatic definition of conflict between belief functions. IEEE Trans. Syst. Man Cybern. B 43(2), 585-596 (2013)

4. George, T., Pal, N.R.: Quantification of conflict in Dempster-Shafer framework: A new approach. International Journal of General Systems 24(4), 407-423 (1996)

5. Höhle, U.: Entropy with respect to plausibility measures. In: Proc. of the $12^{\text {th }}$ IEEE Int. Symposium on Multiple Valued Logic. pp. 167-169. Paris (1982)

6. Hunter, A., Konieczny, S.: Measuring inconsistency through minimal inconsistent sets. In: Proc. of the Eleventh Int. Conf. KR. vol. 8, pp. 358-366. Sydney, Australia (Sept 16-19 2008)

7. Jiroušek, R., Shenoy, P.P.: On properties of a new decomposable entropy of Dempster-Shafer belief functions. International Journal of Approximate Reasoning 119(4), 260-279 (2020) 
8. Klir, G.J., Parviz, B.: A note on the measure of discord. In: Dubois, D. (ed.) Proc. of Eighth Conference on Artificial Intelligence. pp. 138-141. California (1992)

9. Klir, G.J., Ramer, A.: Uncertainty in the Dempster-Shafer Theory: A critical reexamination. International Journal of General Systems 18(2), 155-166 (1990)

10. Klir, G.J., Smith, R.M.: Recent developments in generalized information theory. International Journal of Fuzzy Systems 1(1), 1-13 (1999)

11. Klir, G.J., Yuan, B.: Fuzzy Sets and Fuzzy Logic: Theory and Applications. Prentice Hall International, Upper Saddle River, NJ (1995)

12. Nguyen, N.T.: On entropy of random sets and possibility distributions. In: Bezdek, J.C. (ed.) The Analysis of Fuzzy Information, vol. 1. CRC Press, Boca Raton, FL (1986)

13. Pichon, F., Jousselme, A.L., Ben Abdallah, N.: Several shades of conflict. Fuzzy Sets and Systems 366, 63-84 (Jul 2019). https://doi.org/10.1016/j.fss.2019.01.014

14. Rényi, A.: On measures of entropy and information. In: Proceedings of the 4th Berkeley Symposium on Mathematics, Statistics and Probability. vol. 1, pp. 547561 (1961)

15. Shafer, G.: A mathematical theory of evidence. Princeton University Press, Princeton, N.J. (1976)

16. Shannon, C.E.: A mathematical theory of communication. Bell System Technical Journal 27, 379-423, 623-656 (1948)

17. Smets, P., Kennes, R.: The transferable belief model. Artif. Intell. 66, 191-243 (1994)

18. Smets, P.: The nature of the unnormalized beliefs encountered in the transferable belief model. In: Proc. of the 8th Int. Conf. on Uncertainty in Artificial Intelligence. pp. 292-297. UAI'92, Morgan Kaufmann Publishers Inc., San Francisco, CA, USA (1992)

19. Tsallis, C.: Possible generalization of Boltzmann-Gibbs statistics. Journal of Statistical Physics 52(1-2), 479-487 (1988)

20. Vajda, I., Zvárová, J.: On generalized entropies, Bayesian decisions and statistical diversity. Kybernetika 43(5), 675-696 (2007)

21. Yager, R.R.: Entropy and specificity in a mathematical theory of evidence. International Journal of General Systems 9, 249-260 (1983)

22. Yager, R.R.: On considerations of credibility of evidence. Int. J. Approx. Reason. 7(1/2), 45-72 (Aug/Sep 1992) 